\title{
Bidding Model Based on Balance Programming between Target and Chance
}

\author{
Gang Lu' ${ }^{1, a}$, Fushuan Wen ${ }^{2, b}$ \\ ${ }^{1}$ State Power Economic Research Institute, Beijing 100052, China \\ ${ }^{2}$ College of Electrical Engineering, Zhejiang University, Hangzhou 310027, China \\ alugang.ee@gmail.com, bfushuan.wen@gmail.com
}

Keywords: genetic algorithm; balance programming between goal and chance; bidding strategies

\begin{abstract}
In a pool-based single-buyer electricity market, a Generation Company (GENCO) is required to considering the decision risk when building the optimal bidding strategy due to the stochastic bidding behavior of the rivals. The optimal decision is to maximize the profit while minimizing the risk, however, they are contradicting targets. This paper proposes a new research framework about risk-constrained optimal bidding strategies based on the stochastic programming method, termed as balance programming between target and chance (BPTC). And this method can favor the GENCO to make the stochastic decision in a more rational, flexible, and applicable manner. A genetic algorithm with Monte Carlo simulation is employed to solve the programming model. The effectiveness of the proposed method is shown through a numerical test.
\end{abstract}

\section{Introduction}

Since the power market reformation, the bidding issue of GENCOs has become a focus of researchers all over the world as [1-7]. Many approaches have been developed to solve it, including approaches based on forecasting the market clearing price [1], on the game theory[2], on the estimation of the bidding behavior of rivals[3-5], and on simulating the bidding behavior of GENCOs by artificial intelligence[6]. Among the approaches, the characterization way of rivals' bidding behavior by the stochastic method is applicable to the comparatively mature market with sufficient historical data. Dispersed stochastic distribution in [3], continuous stochastic distribution in [4] and fuzzy theory in [5] are applied to depict rivals' behavior.

Additionally, the bidding strategies of GENCOs are based on incomplete information with absolute risk, so the decision-making risk should be taken into the bidding strategy optimization. Risk is defined as the difference of anticipated and practical profit in [7], and the chance-constrained programming is used to structure the stochastic optimization model. Theoretically, the three stochastic decision-making optimization methods, that is, Expected value model(EVM), Chance-constrained programming $(\mathrm{CCP})$ and Dependent chance programming(DCP), have different inherent preference in mathematical model because of the intrinsic distinction of perspective in the profit and chance, so they are not favourable for the more flexible decision-making between maximizing profit and avoiding risk for decision-makers. Therefore, the same GENCO will attain different and even opposite conclusions with different model.

On the above background, for optimizing the GENCO's bidding strategy, a new research framework with the risk-constrained stochastic decision-making optimization model is proposed in the paper, which is the direct expansion of the literatures [7], and the bidding decision-making risk is defined as the condition in which the practical profit is lower than the anticipated profit. Also in the framework, the paper maximizes the decision-maker's utility with consideration of both profit and risk, based on the overall points in the effective decision frontier curve. Lastly, the simulation case demonstrates the main features of the proposed method. 


\section{Issue Description}

In a pool-based single-buyer electricity market, the pool, acting as a single-buyer, purchases and determines a set of generation outputs from Gencos, according to minimizing the total purchasing cost under meeting the forecasted load demand. Sealed auction and uniform market clearing price (MCP) are applied. Suppose that each Genco bids a linear supply function, and maximizes its profit by choosing the optimal function coefficients. The clearing process can be described as follows:

$$
\begin{array}{cl}
\text { Min } & R \cdot Q \\
& Q(R)=Q_{0}-K R \\
& \sum_{j=1}^{n} P_{j}=Q(R) \\
& \kappa_{j}+\lambda_{j} P_{j}=R \\
\underline{P}_{j} \leq P_{j} \leq \bar{P}_{j} \\
j=1,2, \ldots, n
\end{array}
$$

where $R$ represents the market clearing price; $Q_{0}$ and $K$ are the function coefficients of load model $Q$; $P_{j}$ denotes the output power of the generating unit-j; the linear supply function, $R(P)=\kappa+\lambda P$, denotes the bidding way and $\kappa$ and $\lambda$ are the coefficients; and $\underline{P}$ and $\bar{P}$ are the lower and upper bounding parameters for output power respectively; $n$ is the total number of the generation companies.

The profit of the studied Genco $i$ in a unit time can be described as follows:

$$
\pi(x)=R(x) \cdot P_{i}(x)-C\left(P_{i}(x)\right)
$$

where $C(P)$ is the production cost function; $x$ denotes decision variables, that is, $\kappa$ and $\lambda$. And the $i$-th Genco can fix one of these two coefficients and then determine another one during the optimization procedure.

The bidding coefficients of rivals are random though they can be estimated based on the historical data as stated in [4]. Then, the problem of building an optimal bidding strategy can be formulated as a stochastic optimization problem.

$$
\max \pi(x, \xi)
$$

where $\xi$ represents random variables such as the bidding coefficients of rivals.

\section{Mathematical Model}

Basic Idea. For the optimization problem as (3), suppose $\bar{\pi}$ is a given anticipated goal profit, and $\delta$ is the realization chance of the event $\delta(x)=\operatorname{Pr}\{\pi(x, \xi) \geq \bar{\pi}\}$.

In the stochastic programming, decision-making should follow two rules: (1)With the same realization chance of return, the decision with the maximal return is dominant. (2)With the same goal return, the decision with the maximal realization chance is dominant. Here, effective decisions are defined as the ones that accord with the above two rules simultaneously. The effective decisions may be represented through $\delta$ and $\bar{\pi}$, and all the effective decisions form a line called as Effective Decision Frontier Curve (EDFC). It is known that $\bar{\pi}$ has a negative correlation with $\delta$.

Certainly, it is better that both $\bar{\pi}$ and $\delta$ are greater simultaneously. An option $x^{*}$ dominates another option $x$ if and only if $\bar{\pi}\left(x^{*}\right) \geq \bar{\pi}(x)$ and $\delta\left(x^{*}\right) \geq \delta(x)$. But the goal profit and the realization chance are contradicting multi-objectives. Accordingly, there exists an optimal decision for a decision maker. The EDFC is objective and exclusive for the studied stochastic problem. Thus, the setting of $\delta$ in CCP 
or $\bar{\pi}$ in DCP in advance does specify the exclusive result corresponding to one point in the EDFC. In other words, CCP or DCP selects certain pair of $(\delta, \bar{\pi})$ according to the ahead different preference and hence CCP or DCP may ignore some other decision options in the decision-making process.

Balancing Rules. To select the optimal one among all the effective decisions, the decision-making may be performed based on the following balancing rules.

a. Basic principles. For a decision maker, the expected points in the EFDC are the ones where the increasing velocity of the goal profit is greater than the decreasing velocity of the realization chance, and the ones where the increasing velocity of the realization chance is greater than the decreasing velocity of the goal profit. The explanation is mathematically shown as follows:

- When $\partial^{2} \bar{\pi} / \partial \delta^{2} \leq 0$, i.e., the convex part of the EDFC, the points in the middle of the curve are better than those at the ends.

- When $\partial^{2} \bar{\pi} / \partial \delta^{2} \geq 0$, i.e., the concave part of the EDFC, the points at the ends are better than the ones in the middle.

b. Comparison of changing velocity of Goal and Chance.As stated above, the points in the non-concave part and the ones at the ends are all optional optimal decision. As the two points at the ends are extreme cases, they may be considered separately. And for the convex part of the EDFC, along the axis $\delta$, points where chance increasing is faster than profit decreasing are gradually turning into points where profit decreasing is faster than chance increasing, and accordingly there exists a turning point in this process, where the two changing velocities are equal, that is, the point satisfying $F^{\prime}=\partial \bar{\pi} / \partial \delta=-1$. Actually, this point is a candidate of the optimal decision because it dominates any other point before or after it in the convex part line based on the balancing rules. So the points satisfying $F^{\prime}=-1$ and $F^{\prime \prime}<0$ are all optional ones.

However, the EDFC is of a complex shape possibly and hence may not be differential everywhere. In the following, $y$ denotes the effective decision in the EDFC. Then the value of $\left\{F_{0^{-}}^{\prime}(\bar{\pi}(y), \delta(y))+F_{0^{+}}^{\prime}(\bar{\pi}(y), \delta(y))+2\right\}^{2}$ can be used to estimate the comparison of changing velocity of goal and chance, $\partial \bar{\pi} / \partial \delta$, where $F_{0^{-}}^{\prime}(\bar{\pi}(y), \delta(y))$ and $F_{0^{+}}^{\prime}(\bar{\pi}(y), \delta(y))$ denote respectively the left differential and the right differential of the effective decision point $(\delta(y), \bar{\pi}(y))$. Certainly, the points with the value nearer to zero are better ones.

c. Indifference Decision Curves.Modern theory of utility is an approach to obtain the decision choice under uncertainty. The utility function or the utility curves may be adopted to express the preference of a decision maker in quantity, for example, $F(\bar{\pi}, \delta)=\bar{\pi} \cdot \delta$.

A decision maker may have the same decision utility for the different pairs of $(\delta, \bar{\pi})$, and these $(\delta, \bar{\pi})$ form an indifference decision curve (IDC). The curve is concave because the differential value $\partial \bar{\pi} / \partial \delta$ with the smaller realization chance is greater than that with the larger realization chance, i.e., the second differential $\partial^{2} \bar{\pi} / \partial \delta^{2}$ is negative.

Obviously, the IDCs are a family of curves corresponding to the different utility levels, and the utility increases along with the upper right-hand direction of the curves. Therefore, the optimal decision is the point where the furthest IDC in the upper right-hand direction is tangent or intersectant to the EDFC.

d. Numerical Weight between Goal and Chance. Additionally, the realization chance $\delta$ is in $[0,1]$. The range of goal profit, $\bar{\pi}$, which may be larger or smaller, varies with different cases and may be incomparable in quantity, which affects the weighing of the preference between them. In other words, the pre-preference of profit or chance in CCP and DCP is not objective enough due to ignorance of their numerical relationship.

Numerical weight between the profit goal and the realization chance may be carried out as follows:

$$
l=-\Delta \bar{\pi} / \Delta \delta \quad \text { s.t. } \quad V(\Delta \bar{\pi})=V(-\Delta \delta)
$$


where $V$ represents a decision maker's subjective value judgment about the profit increasing and the corresponding chance decreasing; $l$, named Value Ratio Coefficient (VRC), is the ration between the profit increasing and the corresponding chance decreasing when the value of profit increasing is equal to that of chance decreasing. Besides, $l$ varies much with the different value of $\delta$, and hence a certain set of VRCs $\left\{l_{i}, \mathrm{i}=1,2, \ldots, n\right\}$ should be adopted, which can be expressed as a function $l=L(\delta)$, where $l_{i}$ stands for the VRC with the range of $\left(\delta_{i-1}, \delta_{i}\right]$ and $n$ is the total number.

The EDFC is modified according to VRC $l$ as follows:

$$
\bar{\pi}^{\prime}(\delta)=\bar{\pi}(\delta) / L(\delta)
$$

So, the points satisfying $\partial \bar{\pi}^{\prime} / \partial \delta=\partial \bar{\pi} /[L(\delta) \cdot \partial \delta]=-1$ in the modified EDFC are the candidates of the best decison, i.e. the points satisfying $F^{\prime}=\partial \bar{\pi} / \partial \delta=-L(\delta)$ in the original EDFC are the ones. Accordingly, the value of $\left\{F_{0^{-}}^{\prime}(\bar{\pi}(y), \delta(y))+F_{0^{+}}^{\prime}(\bar{\pi}(y), \delta(y))+2 L(\delta)\right\}^{2}$ is applied to estimate the comparison of changing velocity of goal and chance, $\partial \bar{\pi} / \partial \delta$, based on the principle that the points with the value nearer to zero are better ones.

Balancing Rules. Based on the BPGC method, the GENCO should maximize the decision utility through comparing the potential anticipated goal $\bar{\pi}$ and the potential realization chance $\delta$ of each decision and referring to the comparison of changing velocity of them along EDFC at the same time. The process can be expressed mathematically as follows:

$$
\begin{cases}\max & \frac{F(\bar{\pi}(y), \delta(y))}{\left\{F_{0^{-}}^{\prime}(\bar{\pi}(y), \delta(y))+F_{0^{+}}^{\prime}(\bar{\pi}(y), \delta(y))+2 L(\delta(y))\right\}^{2 p}+q} \\ \text { s.t. } & y \in\left\{x \mid \delta(x, \bar{\pi}(x)) \geq \delta\left(x^{\prime}, \bar{\pi}(x)\right), \forall x^{\prime} \in X\right\} \\ & \delta(x)=\operatorname{Pr}\{\pi(x) \geq \bar{\pi}(x)\} \geq \delta_{0} \\ & \delta(x) \in(0,1] \\ & \bar{\pi}(x) \geq \pi_{0} \\ & x \in X\end{cases}
$$

where $F$ represents a decision utility function; and $x$ is the decision vector; and $y$ denotes the effective decisions in the EDFC; and $F^{\prime}$ is the partial differential $\partial \bar{\pi} / \partial \delta$ of the points in the EDFC, in which $F_{0^{-}}^{\prime}(\bar{\pi}(y), \delta(y))$ and $F_{0^{+}}^{\prime}(\bar{\pi}(y), \delta(y))$ are the left differential and the right differential of the point ( $\delta(y), \bar{\pi}(y))$ respectively; and $L(\delta)$ is the VRC function; and $\delta_{0}$ is the minimum chance accepted; and $\pi_{0}$ is the minimum goal accepted; and $p$ and $q$ are non-minus coefficients and $p$ is generally fixed as an integer.

For a decision maker, the functions, $F$ and $L(\delta)$, and the coefficients, $\delta_{0}$ and $\pi_{0}$, embody the decision-making modal of personal preference, while comparison of changing velocity of goal and chance is a kind of objective decision-making way. Accordingly, the BPGC can take the decision maker's personal preference information and the objective and overall information of the problem into consideration as a whole. Actually, the coefficients, $p$ and $q$, show the subjective degree of the decision way, as the equation $p=0$ means that the decision-making process is totally subjective on one hand and the subjective degree is stronger with bigger $q$ on the other hand. Additionally, the equation $F=1$ means that the decision utility is ignored; however this way may generate several feasible solutions. Hence, both the objective and subjective decision way need be integrated to get the exclusive optimal decision. 


\section{Proposed Genetic Algorithm Solution Procedure}

A GA with the Monte Carlo method is developed to solve the BPGC as given in (6). The real coding scheme with the form of $[\mathrm{x}-, \mathrm{x}, \mathrm{x}+]$ is adopted, in which $\mathrm{x}-, \mathrm{x}$, and $\mathrm{x}+$ are all decision variables. The details are as follows:

- Population, maximal generation, crossover probability, mutation probability, and sampling times $M$ are 80, 100, 0.9, 0.005 and 5000, respectively.

- The realization chance, $\delta_{i}=0.45+0.009 *(\mathrm{i}-1), i=1,2, \ldots, 61$, that is, $\delta_{i} \in[0.45,0.99]$.

- Fitness calculation. Rank the $\pi$ obtained from the $M$ simulation results for each individual in a descending order and designate the $\pi$ in the $\left\lceil\delta_{i} \cdot M\right\rceil$ place as the $\bar{\pi}_{i}$ corresponding to $\delta_{i}$, where symbol $\lceil\cdot\rceil$ denotes the upward rounding operator. Then get $n$ pairs of $\left(\bar{\pi}_{i}, \delta_{i}\right),\left(\bar{\pi}, \delta_{i}-\Delta \delta\right)$ and $\left(\bar{\pi}, \delta_{i}+\Delta \delta\right)$ for every individual. And then the following formula is adopted to calculate the fitness of every individual:

$$
\begin{aligned}
& \max \frac{F[\delta, \bar{\pi}(\delta, x)]}{\left\{\left[\bar{\pi}\left(\delta+\Delta \delta, x^{-}\right)-\bar{\pi}\left(\delta-\Delta \delta, x^{+}\right)\right] / \Delta \delta+2 L(\delta(x))\right\}^{-2 p}+q}+ \\
& F\left[\delta-\Delta \delta, \bar{\pi}\left(\delta-\Delta \delta, x^{-}\right)\right]+F\left(\delta+\Delta \delta, \bar{\pi}\left(\delta+\Delta \delta, x^{+}\right)\right)
\end{aligned}
$$

where the parameter $\Delta \delta$ is assumed as 0.002 ; and the value of $q$ turns from 0.4 to 0.01 along evolution, which can control the optimizing process from the initial quick approach to the curve EDFC.

\section{Numerical Case And Result Analysis}

Case. The numerical example in [7] is directly employed. There are six Gencos participating in an electricity market, and their production cost function $\mathrm{C}(\mathrm{P})$ is assumed as $C(p)=a_{1} p+0.5 a_{2} p^{2}$, and in the linear supply function of the rivals, the coefficients $\kappa$ and $\lambda$ are supposed to obey the normal distribution with the correlation coefficient equating -0.1 , whose expected values and variance deviations are following:

$$
\left\{\begin{array} { l } 
{ \mu ( \kappa ) = 1 . 2 a _ { 1 } } \\
{ \mu ( \lambda ) = 1 . 2 a _ { 2 } }
\end{array} \left\{\begin{array}{l}
\sigma(\kappa)=0.15 a_{1} / 4 \\
\sigma(\lambda)=0.15 a_{2} / 4
\end{array}\right.\right.
$$

The coefficients of production cost function and the limits on the output power are shown in Table 1 , taken from [7]. Here, the generation company-2 is studied and its bidding coefficient $\kappa_{2}$ is fixed at 1.75 , i.e., the decision variable is $\lambda_{2}$. The coefficients $Q_{0}$ and $K$ of the load model function are 450 and 20 respectively.

Table 1. Production cost function coefficients and output power limits.

\begin{tabular}{ccccc}
\hline Unit No. & $a_{1}$ & $a_{2}$ & $\underline{P}(\mathrm{MW})$ & $\bar{P}(\mathrm{WM})$ \\
\hline 1 & 2 & 0.00875 & 50 & 160 \\
2 & 1.75 & 0.035 & 50 & 100 \\
3 & 1 & 0.0625 & 30 & 80 \\
4 & 3.15 & 0.00334 & 30 & 80 \\
5 & 3 & 0.015 & 10 & 60 \\
6 & 3 & 0.015 & 10 & 60 \\
\hline
\end{tabular}

The adopted utility function is as follows:

$$
F=[\bar{\pi}(x)]^{h} \cdot \delta(x)
$$

where $h$ denotes the preference index of the generation company. 
The parameters, $\delta_{0}, f_{0}, p$ and $q$, are assumed as $0.5,65,1$ and 0.01 respectively. The VRC is assumed as follows:

$$
l=L(\delta)=\left\{\begin{array}{cc}
40 & \delta \in(0.45,0.8] \\
30 & \delta \in(0.8,1.0]
\end{array}\right.
$$

Numerical Results. The EDFC based on CCP are firstly shown as the convex real line in Fig. 1. When p equals 0 , the impact of different preference indices $h$ on the decision results is analyzed as follows. The higher value of $h$ shows, the generation company prefers to the goal profit more. When $h$ equals 1, 2 and 5 respectively, the best decision points are the tangent to the points P1, P2 and P3 respectively between the EDFC and the IDC I, II and III. As $h$ is increasing, the IDC inclines to the left and the optimal point also tends to the left, and thus, the return becomes large with the decreasing realization chance. The relevant results of the above three optimal points are listed in Table 2.

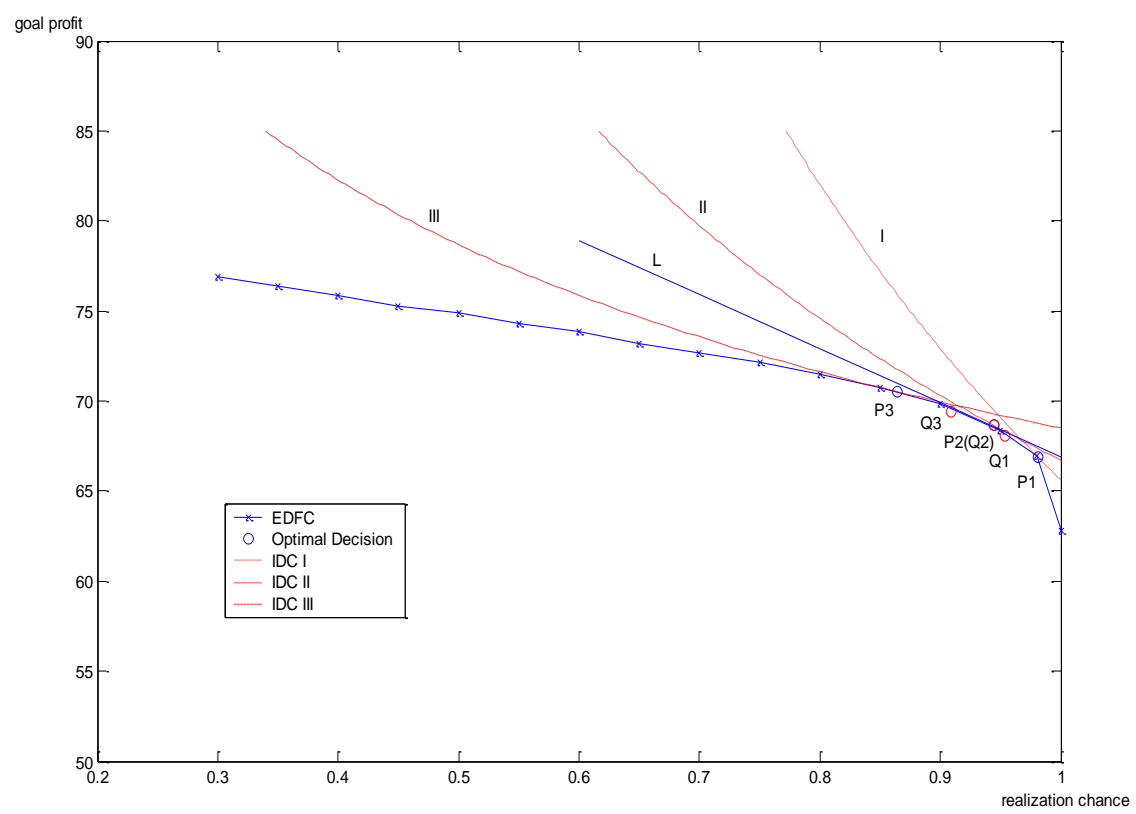

Fig. 1. Relation chart between Goal Profit and Realization Chance

When $p$ equals $1, h$ equals 1, 2 and 5 respectively, the best decision points are points Q1, Q2 and Q3 respectively. The line $L$ in Fig. 1 is tangent to the EDFC with the slope of -30 . When comparison of changing velocity of goal and chance is considered, the best points turn from points P1, P2 and P3 into points Q1, Q2 and Q3. It can be thought that the best decision point is "attracted" by the tangent point between the line $L$ and the EDFC, that is, the best one is obtained from weight between the tangent point and the IDC. The process takes both the generation company's subjective preference and the objective condition of the bidding problem into consideration. Besides, as $q$ is very small, the impact of comparison of changing velocity is great, and hence Q3 deviates from P3 remarkably.

If DCP is used to build the bidding strategy, the anticipated goal profit is required to be set in advance, i.e., one point in the axis $\bar{\pi}$ is selected in advance and the corresponding point in the EDFC is taken as the decision result. Similarly, if CCP in [7] is adopted, the expected realization chance is required to be set in advance, i.e., one point in the axis $\delta$ is selected in advance and the corresponding point in the EDFC is taken as the decision result. But for the convex shape of the EDFC, it is known that the turning part of the line nearly between points P1 and P3 includes better solutions. The generation company could choose a so-called best point in this range by making a compromise among several considerations. Using the indirect analysis, BPGC could reflect the cognition process of the decision maker more objectively and comprehensively in the stochastic problems, and hence the outcome will be more rational. 
Table 2. The Optimal Bidding of the Generation Company

\begin{tabular}{ccccc}
\hline$p$ & $h$ & $\bar{\pi}$ & $\delta$ & $x$ \\
\hline \multirow{3}{*}{0} & 1 & 66.8904 & 0.981 & 0.03704 \\
& 2 & 68.6295 & 0.945 & 0.03703 \\
& 5 & 70.5319 & 0.864 & 0.03699 \\
\hline \multirow{2}{*}{1} & 1 & 68.0867 & 0.954 & 0.03774 \\
& 2 & 68.6295 & 0.945 & 0.03703 \\
& 5 & 69.403 & 0.909 & 0.03690 \\
\hline
\end{tabular}

Based on the above case analysis and the mathematical theory, it is known that the bidding method has the following features: The concept of the EDFC can supply the decision makers with all the effective decision points $(\delta, \bar{\pi})$. Accordingly, the decision process has enough flexibility. And the shape of the curve may give the decision maker comprehensive guidance, so BPGC can obtain the optimal results, while CCP and DCP may overlook the whole information of the EDFC and then get a sub-optimal decision, on the contrary.

\section{Conclusion}

Based on the stochastic programming model, termed as Balance Programming between Goal and Chance (BPGC), a new framework is developed in this paper for building optimal bidding strategies with considering the risk for the generation companies. BPGC is favor for the decision makers to adopt more suitable decision-making strategy through balancing goal and chance based on the all-around comprehension of the real problem. Simulation results have shown the rational, flexible, and applicable manner of this method. How to include those factors such as the unit physics constraints and other bidding way into the research framework is our future work.

\section{References}

[1] A.J. Conejo, F.J. Nogales, J.M Arroyo, Price-taker bidding strategy under price uncertainty. IEEE Trans on Power System, vol.17, no. 4, 1081-1088 (2002)

[2] J. Contreras, O. Candiles, J.I. de la Fuente, et al, A cobweb bidding model for competitive electricity markets. IEEE Trans on Power Systems, vol. 17, 148-153 (2002)

[3] H.L. Song, C.C. Liu, J. Lawarree, Optimal electricity supply bidding by Markov decision process. IEEE Trans on Power Systems, vol.15, no.2, 618-624 (2000)

[4] F. Wen, A.K. David, Optimal bidding strategies and modeling of imperfect information among competitive generators. IEEE Trans on Power Systems, vol. 16, 15-21 (2001)

[5] C. Mattson, D, Lucarella, C. C. Liu, Modeling a competitor's bidding behavior using fuzzy inference networks. Proc. International Conference on Intelligent System Applications in Power Systems, Budapest, Hungary, pp.1117-1130 (2001)

[6] G.R. Gajjar, S.A. Khaparde, P.P. Nagaraju, et al, Application of actor-critic learning algorithm for optimal bidding problem of a Genco. IEEE Trans on Power Systems, vol.18, no.1, 11-18 (2003)

[7] Xinshun Ma, Fushuan Wen, Jianxin Liu, A chance constrained programming based approach for building optimal bidding strategies for generation companies with risk management. Power System Technology, vol. 29, no. 10, 35-39 (2005) 